\title{
Hepatic Iron Deprivation Prevents Spontaneous Development of Fulminant Hepatitis and Liver Cancer in Long-Evans Cinnamon Rats
}

\author{
Junji Kato, ${ }^{\star}$ Masayoshi Kobune, ${ }^{*}$ Yutaka Kohgo, Naoki Sugawara,, Hiroyuki Hisai, ${ }^{\star}$ Tokiko Nakamura, ${ }^{\star}$ Sumio Sakamaki, \\ Norimasa Sawada, ${ }^{\S}$ and Yoshiro Niitsu* \\ *Fourth Department of Internal Medicine, ${ }^{\ddagger}$ Department of Public Health, and ${ }^{\S}$ Second Department of Pathology, Sapporo Medical \\ University School of Medicine, Sapporo 060; and "Third Department of Internal Medicine, Asahikawa Medical College, \\ Asahikawa 078, Japan
}

\begin{abstract}
Several clinical studies have suggested that excess hepatic iron accumulation is a progressive factor in some liver diseases including chronic viral hepatitis and hemochromatosis. However, it is not known whether iron-induced hepatotoxicity may be directly involved in hepatitis, cirrhosis, and liver cancer. The Long-Evans Cinnamon (LEC) rat, which accumulates excess copper in the liver as in patients with Wilson's disease, is of a mutant strain displaying spontaneous hemolysis, hepatitis, and liver cancer. We found previously that LEC rats harbored an additional abnormality: accumulation of as much iron as copper in the liver. In the present study, we compared the occurrence of hepatitis and liver cancer in LEC rats fed an iron-deficient diet (ID) with those in rats fed a regular diet (RD). The RD group showed rapid increments of hepatic iron concentrations as the result of hemolysis, characteristics of fulminant hepatitis showing apoptosis, and a $53 \%$ mortality rate. However, no rats in the ID group died of fulminant hepatitis. Hepatic iron, especially "free" iron concentration and the extent of hepatic fibrosis in the ID group were far less than those of the RD group. At week 65, all rats in the RD group developed liver cancer, whereas none did in the ID group. These results suggest that the accumulation of iron, possibly by virtue of synergistic radical formation with copper, plays an essential role in the development of fulminant hepatitis, hepatic fibrosis, and subsequent hepatocarcinogenesis in LEC rats. (J. Clin. Invest. 1996. 98:923-929.) Key words: iron overload - hepatitis • hepatoma • iron depletion • apoptosis
\end{abstract}

\section{Introduction}

Some clinical studies have suggested that excess hepatic iron accumulation is a progressive factor in some chronic liver diseases including chronic viral hepatitis and hemochromatosis

Address correspondence to Dr. Yoshiro Niitsu, Fourth Department of Internal Medicine, Sapporo Medical University School of Medicine, South-1, West-16, Chuo-ku, Sapporo 060, Japan. Phone: 81-11611-2111x3260; FAX: 81-11-612-7987; E-mail: niitsu@sapmed.ac.jp

Received for publication 13 March 1996 and accepted in revised form 20 June 1996.

J. Clin. Invest.

(C) The American Society for Clinical Investigation, Inc.

0021-9738/96/08/0923/07 \$2.00

Volume 98, Number 4, August 1996, 923-929
(1-3). It has been shown that iron reduction by phlebotomy significantly improved the liver damage in patients with hemochromatosis (4) and chronic hepatitis C (5). However, it has not been clarified whether iron-induced hepatotoxicity is directly involved in hepatitis, cirrhosis, or liver cancer.

The Long-Evans Cinnamon (LEC) ${ }^{1}$ rat has been established from a closed colony of Long-Evans rats. LEC rats suffer from spontaneous hepatitis with hemolytic anemia and jaundice developing around 4 mo after birth, followed by death in $50 \%$ of rats due to fulminant hepatitis $(6,7)$. The rats which recovered from the fulminant hepatitis exhibited chronic hepatitis and cholangiofibrosis, and a subsequent high rate of liver cancer (8). LEC rats accumulate excess copper in the liver, but have decreased levels of serum ceruloplasmin activities, a clinical presentation similar to human Wilson's disease (WD) $(9,10)$. Genetic analysis demonstrated that $h t s$ was a responsible gene for hepatitis in LEC rats (11), and recent cloning analyses revealed that both $h t s$ and the human WD gene were homologous genes encoding rat and human novel P-type adenosine triphosphatase (ATPase), respectively (12). In both LEC rats and WD patients, absence or dysfunction of the P-type ATPase results in impairment of copper incorporation into newly synthesized ceruloplasmin in hepatocytes $(12,13)$.

On the other hand, we demonstrated previously that LEC rats accumulated as much iron as copper in the liver and brain $(14,15)$ and had elevated serum transferrin levels, an abnormality in iron metabolism. Therefore, we have postulated that excess hepatic iron accumulation, in addition to copper accumulation, contributes to the development of the liver injury in LEC rats. Free metal ions such as copper and iron are known to be important for the production of free radicals, among which iron is most potent in vivo $(16,17)$. The generated free radicals induce lipid peroxidation, DNA breakage, and 8-hydroxydeoxy guanosine formation, resulting in tissue damage and DNA mutagenesis $(18,19)$.

In patients with WD, liver dysfunction after hemolytic anemia was sometimes observed as an initial clinical manifestation similar to LEC rats $(20,21)$. As to the cause of hemolysis in WD patients, it was suggested that increased circulation of inorganic copper ions damaged erythrocyte membranes $(22,23)$. Thus, a possible increase of hepatic iron influxed from lysed erythrocytes may contribute to the liver damage in LEC rats.

In this study, by comparing the occurrence of hepatitis and liver cancer in LEC rats fed an iron-deficient diet (ID) with the

1. Abbreviations used in this paper: ALT, alanine aminotransferase; $\mathrm{Hb}$, hemoglobin; ID, iron-deficient diet; LDH, lactate dehydrogenase; LEC rat, Long-Evans Cinnamon rat; RD, regular diet; WD, Wilson's disease. 


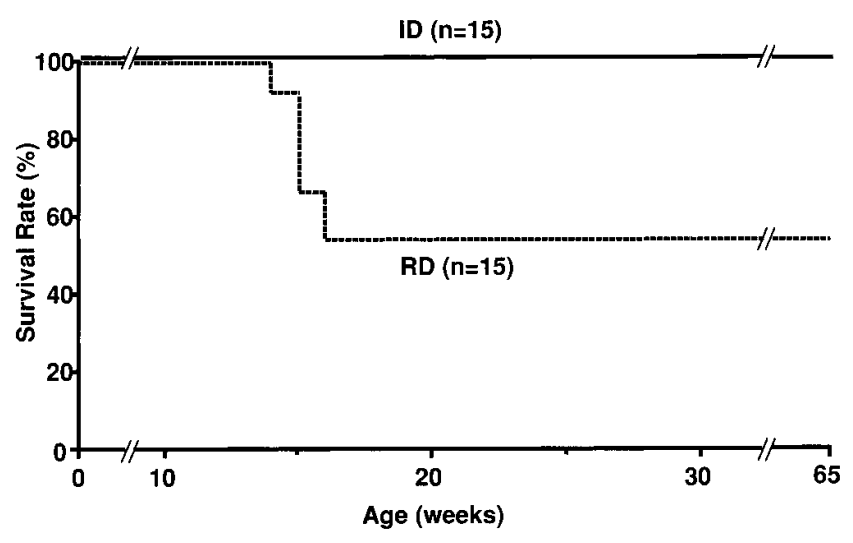

Figure 1. Survival curves of LEC rats fed with the RD or ID diet. Two groups of 15 rats were fed with RD or ID from 6 to $65 \mathrm{wk}$ after birth. 8 of the 15 rats in the RD group died of fulminant hepatitis between 14 and $16 \mathrm{wk}$, whereas all the rats in the ID group survived the observation period.
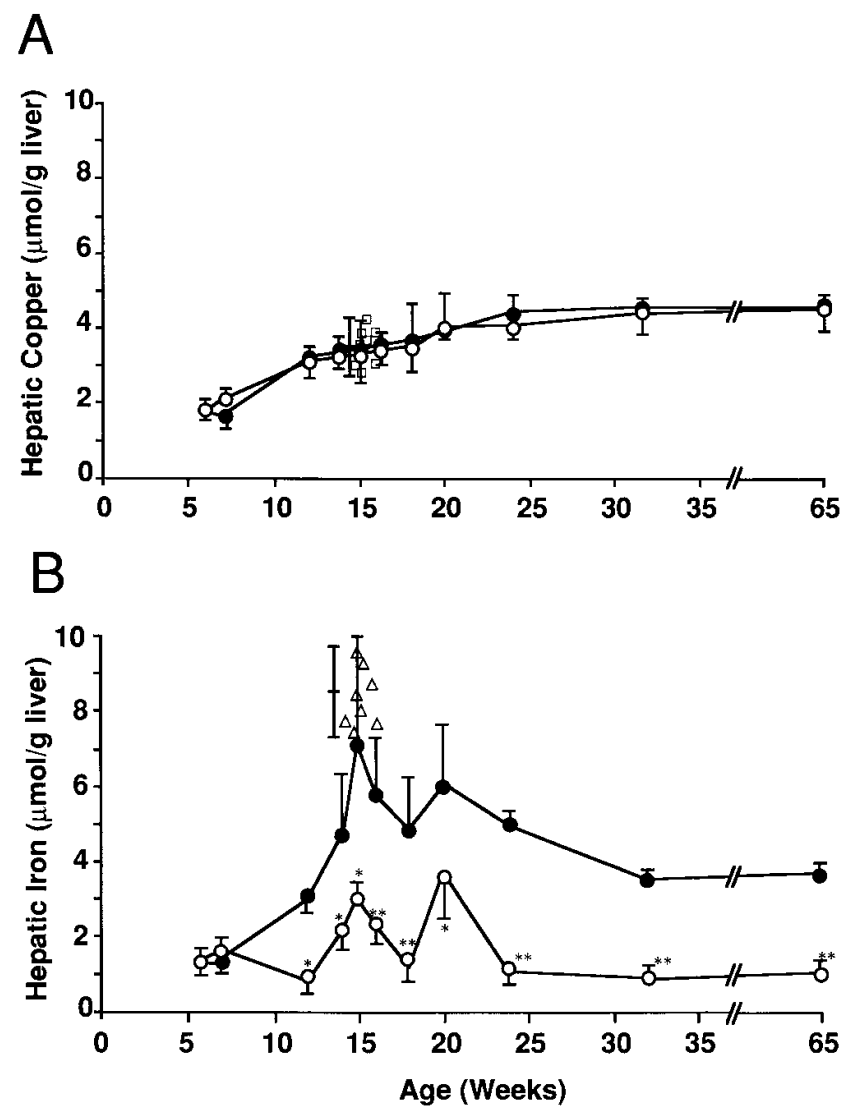

Figure 2. Serial changes of hepatic copper and iron levels in LEC rats. The rats were fed with RD (filled circles) or ID (open circles), five rats in each group were killed at the indicated age, and hepatic levels of copper $(A)$ and iron $(B)$ were determined by using atomic absorption technique. Hepatic copper (open squares) and iron (open triangles) levels of 8 of the 33 rats which died of fulminant hepatitis were also measured. Each error bar represents the mean $\pm 1 \mathrm{SD}$, of five rats. $* P<0.05$ vs. $\mathrm{RD} ; * * P<0.01$ vs. $\mathrm{RD}$.
Table I. Concentrations of Nonheme and Bleomycin-detectable Iron in the Livers of LEC Rats

\begin{tabular}{llllll}
\hline & \multicolumn{2}{c}{$\mathrm{RD}$} & & \multicolumn{2}{c}{$\mathrm{ID}$} \\
\cline { 2 - 3 } \cline { 5 - 6 } Group & $7 \mathrm{wk}$ & $15 \mathrm{wk}$ & & $7 \mathrm{wk}$ & $15 \mathrm{wk}$ \\
\hline $\begin{array}{c}\text { Nonheme iron } \\
(\mu \mathrm{mol} / \text { gram })(n=5)\end{array}$ & $0.7 \pm 0.2$ & $5.4 \pm 1.4^{* *}$ & $0.6 \pm 0.2$ & $2.0 \pm 0.4^{\ddagger}$ \\
$\begin{array}{c}\text { Bleomycin-detectable } \\
\text { iron }(\mu \mathrm{mol} / \text { gram })(n=5)\end{array}$ & $0.2 \pm 0.0$ & $1.2 \pm 0.3^{\sharp \ddagger}$ & $0.2 \pm 0.0$ & $0.5 \pm 0.2$
\end{tabular}

Data are expressed as means \pm 1 SD. $* P<0.01$ compared with group receiving ID at $15 \mathrm{wk} .{ }^{\S} P<0.05$ compared with group receiving ID at 15 wk. ${ }^{\ddagger} P<0.01$ compared with group at $7 \mathrm{wk}$.

occurrence in rats fed a regular diet (RD), we investigated if excess iron in addition to copper accumulation may contribute to the development of hepatitis and hepatoma.

\section{Methods}

Animals. Male LEC rats were provided from the Center for Experimental Plants and Animals of Hokkaido University and were maintained at Sapporo Medical University under conventional conditions. The rats were fed with either the RD or ID diet (Oriental Kohbo Co., Tokyo, Japan). In the diets, the RD and ID contain ferric ammonium citrate of $12.3 \mathrm{mg} / 100$ grams and $0.03 \mathrm{mg} / 100$ grams, respectively, and
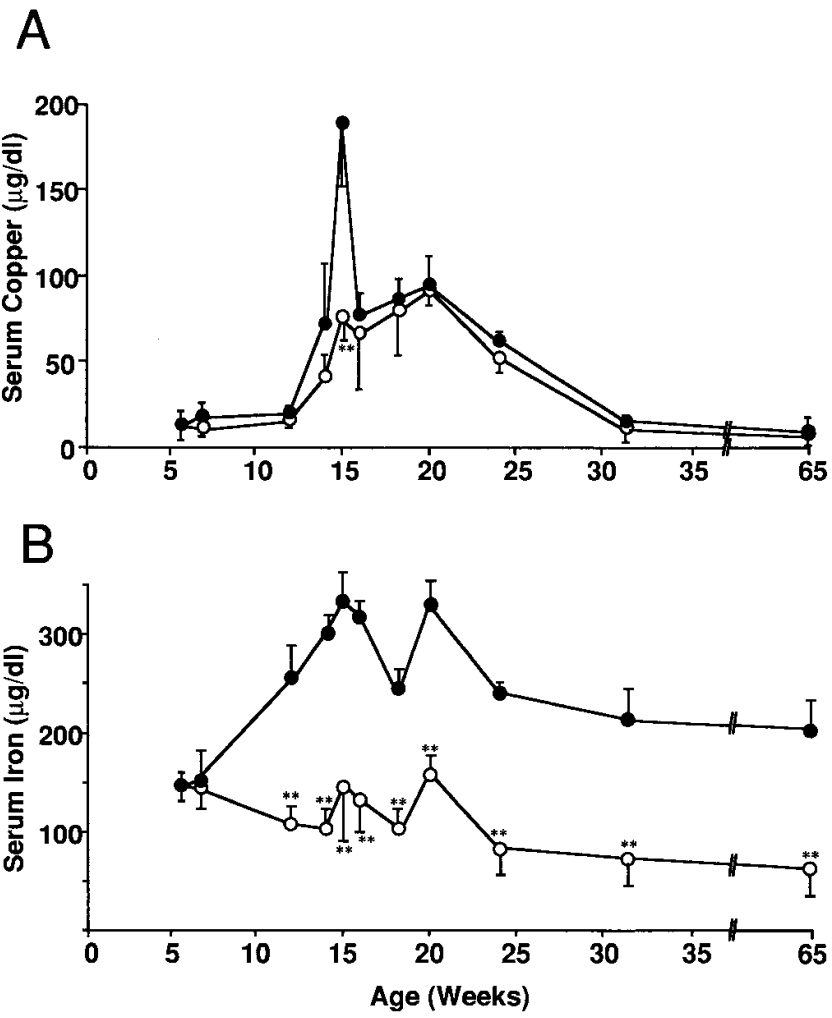

Figure 3. Serial changes of serum copper and serum iron concentrations. The rats were fed with RD (filled circles) or ID (open circles), five rats in each group were killed at the indicated age, and serum levels of copper $(A)$ and iron $(B)$ were determined. Each error bar represents the mean $\pm 1 \mathrm{SD}$, of five rats. $* P<0.05$ vs. RD; $* * P<0.01$ vs. RD. 


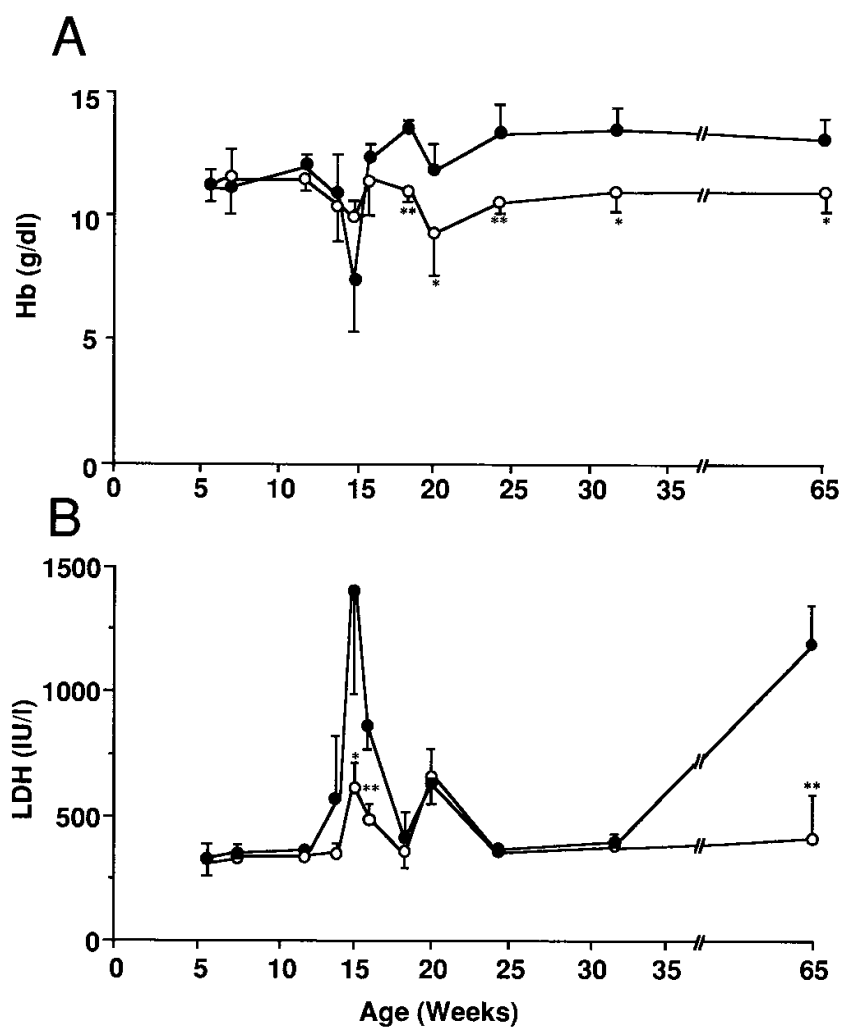

Figure 4. Serial changes of blood $\mathrm{Hb}$ concentration and serum LDH activity. The rats were fed with RD (filled circles) or ID (open circles), five rats in each group were killed at the indicated age, and blood $\mathrm{Hb}(A)$ and serum LDH activity $(B)$ were determined. Each error bar represents the mean $\pm 1 \mathrm{SD}$, of five rats. $* P<0.05 \mathrm{vs.} \mathrm{RD;}$ $* * P<0.01$ vs. RD.

the other contents, including copper, carbohydrate, vitamins, amino acids, minerals, and lipids, were equilibrated. Double-distilled water was given to the rats via plastic bottles to prevent iron contamination. The experiments were humanely conducted in accordance with the recommendations of the Guide for the Care and Use of Laboratory Animals of Sapporo Medical University School of Medicine.

Determination of hepatic iron and copper. All glassware was acidwashed and dried to prevent iron and copper contamination. Rats were anesthetized, the portal vein was cannulated with a 21-gauge Teflon catheter, and to prevent contamination of hemoglobin iron, the liver of each rat was perfused with $50 \mathrm{ml}$ of $0.9 \% \mathrm{NaCl}$ to wash out the blood. The liver was then resected by stainless scissors, and the total iron and copper concentrations of the liver were determined by using an atomic absorption spectrophotometer with an air/acetylene flame (type 208; Hitachi Kohki Co., Tokyo, Japan) as described previously (14). Nonheme iron concentrations in the liver were measured by the 2,2'-bipyridyl method (24) and free iron in the liver was estimated by measuring the bleomycin-detectable iron in the tissue according to the method of Baliga et al. (25).

Determination of serum concentrations of bilirubin, lactate dehydrogenase ( $L D H)$, iron, copper, alanine aminotransferase $(A L T)$, and blood hemoglobin $(\mathrm{Hb})$. Blood was taken from the jugular vein of each rat killed. Serum concentrations of total bilirubin, direct bilirubin, LDH, and ALT were determined by using automatic biochemical analyzer (model 736-30; Hitachi, Tokyo, Japan). Blood Hb concentration was determined using the cyanmethemoglobin method (26). Serum iron concentration was measured by using Quick auto Neo Fe kit (Shino Test Co., Tokyo, Japan) based on a colorimetric method using 2-nitroso-5-( $N$-propyl- $N$-sulfopropylamino)phenol as
A
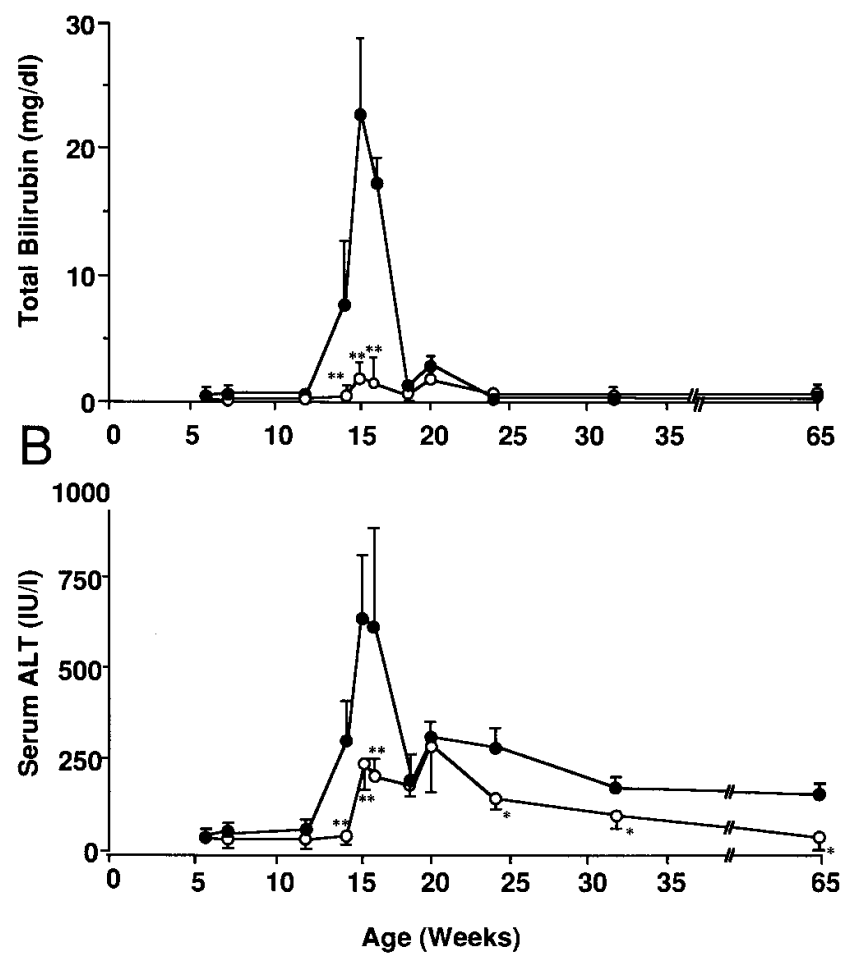

Figure 5. Serial changes of total bilirubin and serum ALT activity. The rats were fed with RD (filled circles) or ID (open circles), five rats in each group were killed at the indicated age, and total bilirubin $(A)$ and serum ALT activity $(B)$ were determined. Each error bar represents the mean $\pm 1 \mathrm{SD}$, of five rats. $* P<0.05$ vs. $\mathrm{RD} ; * * P<0.01$ vs. RD.

the chromogen (27). Serum copper concentration was determined according to the method of Makino and Itoh (28).

Histological examination. A portion of rat liver was removed and fixed in $10 \%$ buffered formalin. Paraffin-embedded sections were stained with hematoxylin and eosin, Gitter, or arusian blue and examined histologically by light microscopy.

Detection of apoptotic cells in the liver section. To detect cell apoptosis, the sections were stained by using an In Situ Cell Death Detection kit, POD (Boehringer Mannheim GmbH, Mannheim, Germany) based on in situ tailing and nick translation techniques (29), and were observed by fluorescence microscopy (Axiovert 405M; Zeiss, Oberkochen, Germany).

Statistical analysis. Data are presented as means \pm 1 SD. Comparisons both within a group and between two groups were made using paired Student's $t$ test. Values were considered to be statistically significant when $P<0.05$.

\section{Results}

Survival curves. Each group of 15 LEC rats was fed with RD (RD group) or ID (ID group) from week 6 and the survival curves of both groups were monitored. As shown in Fig. 1, $53 \%(8 / 15)$ of the rats in the RD group died of fulminant hepatitis around weeks $14-16$, and the remaining rats survived until they were killed at week 65 . None of the rats in the ID group died until they were killed at week 65 .

Hepatic copper and iron levels. To determine serial changes of hepatic copper and iron levels, 83 rats were fed with RD and 

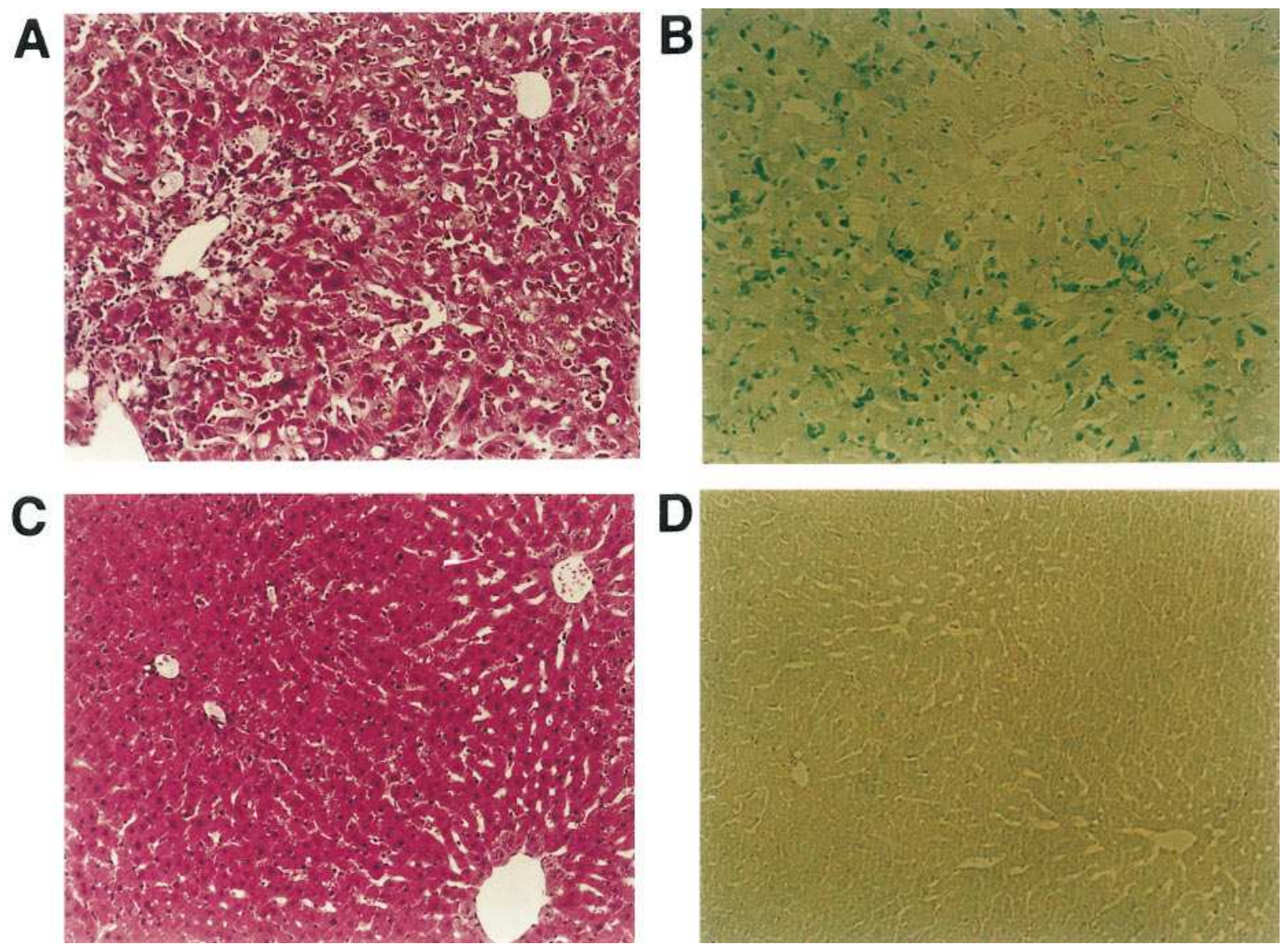

Figure 6. Histological findings of livers obtained from rats fed with $\mathrm{RD}(A$ and $B)$ or ID $(C$ and $D)$ at 15 wk after birth. The liver sections were stained with hematoxylin and eosin $(A$ and $C)$ and arusian blue $(B$ and $D) . \times 100$.

50 rats with ID after week 6 when 5 rats were killed to obtain pretreatment values, and thereafter 5 rats in each group were killed at weeks 7, 12, 14, 15, 16, 18, 20, 24, 32, and 65. In the RD group, 33 rats died of fulminant hepatitis between weeks 14 and 16, whereas all the rats in the ID group survived the observation period (week 65).

Hepatic copper levels of the rats killed in both groups showed similar accumulation curves: a gradual twofold increment reaching a plateau at week 24. Mean hepatic copper level in rats which died of fulminant hepatitis $(3.2 \pm 0.6 \mu \mathrm{mol} / \mathrm{gram}$ liver) was almost the same as in the rats killed at week 15 (3.2 $\pm 0.7 \mu \mathrm{mol} /$ gram liver) (Fig. $2 A$ ).

Hepatic iron levels of the rats killed in the RD group rapidly increased from week 12, and thereafter were remarkably elevated, forming two peaks at week 15 (sixfold of the level at week 6) and at week 20 (fivefold of the level at week 6). Mean hepatic iron level in rats which died of fulminant hepatitis was $8.6 \pm 1.6 \mu \mathrm{mol} / \mathrm{gram}$ liver, which was apparently even higher, though not statistically significant, due to the limited number of cases examined, than that of rats killed at week $15(7.2 \pm 2.9$ $\mu \mathrm{mol} /$ gram liver) (Fig. $2 \mathrm{~B}$ ). As shown in Fig. $4 \mathrm{~A}$, depressions of $\mathrm{Hb}$ concentrations reciprocal to the two peaks of hepatic iron increments were observed in the RD group, indicating that iron derived from erythrocyte rapidly influxed to the liver after hemolysis. Moreover, the hepatic iron decreased corresponding to the recovery of $\mathrm{Hb}$, possibly due to mobilization of the iron into bone marrow.
Hepatic iron levels of the rats killed in the ID group showed markedly low levels throughout the observation period, suggesting that hepatic iron was effectively depleted.

We then measured nonheme and bleomycin-detectable iron concentrations in the livers. The hepatic nonheme iron levels of the rats killed in the RD group were significantly higher than those in the ID group at week 15 (Table I). We found a significant increase in the bleomycin-detectable iron level in the liver of RD group at week 15 (Table I). Therefore, it is suggested that the increased hepatic iron, especially nonheme iron including free iron, contributed more to the liver damage in the RD group.

Serial changes of serum iron, copper, LDH, bilirubin, ALT, and blood $\mathrm{Hb}$. Because we could not obtain blood samples from the 33 dead rats in the RD group, serum iron, copper, $\mathrm{LDH}$, bilirubin, ALT, and blood $\mathrm{Hb}$ concentrations were determined in the rats that were killed. As shown in Fig. $3 A$, serum copper in both groups from weeks 6-12 were markedly low compared with that of Wistar rats $(100 \pm 20 \mu \mathrm{g} / \mathrm{dl})$. However, the serum copper levels drastically increased during the period of weeks 12-20, and then decreased after week 24 in both groups. The maximum level at week 15 in the RD group was significantly higher than that in the ID group $(P<0.01)$, suggesting that more copper was released as the result of fulminant hepatitis. The serum copper levels in both groups showed gradual decreases after week 20, compatible with the previous finding that the synthesis of metallothionein was sufficiently induced to chelate copper after this period (30). 

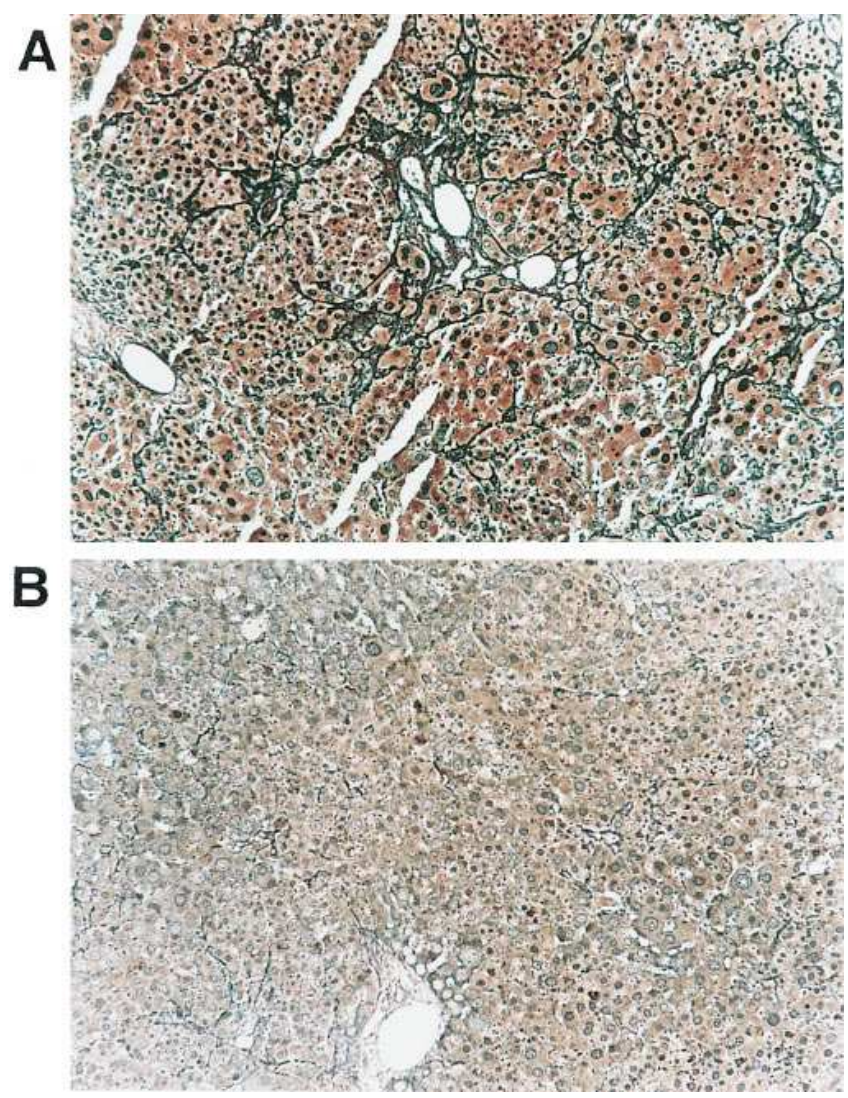

Figure 7. Fibrosis in the livers obtained from rats fed with $\mathrm{RD}(A)$ or ID $(B)$ at $32 \mathrm{wk}$ after birth. The liver sections were stained with Gitter. $\times 200$.

Serum iron concentrations in the RD group rapidly elevated immediately after birth to form a peak at week 15 , and then a transient depletion again followed a peak at week 20 which gradually became a plateau maintaining relatively high levels, suggesting that iron absorption is increased in LEC rats (Fig. $3 \mathrm{~B}$ ). These two peaks of increased serum iron levels were considered to be the result of hemolysis, because serum iron consists of transferrin-bound iron and $\mathrm{Hb}$-bound iron. In contrast, serum iron in the ID group showed markedly low levels throughout the observation period.

$\mathrm{Hb}$ concentration in both RD and ID groups showed two transient depletions at weeks 15 and 20, although the degree of depletion was much greater in the RD group. In the ID group, $\mathrm{Hb}$ levels were significantly lower than those of the RD group after week 18, reflecting the iron-deficient state. The serum LDH activities showed a reciprocal pattern of $\mathrm{Hb}$ concentration at weeks 15 and 20, but between weeks 32 and 65 they exhibited an increase of LDH without hemolysis, probably due to the development of liver cancer (31) (Fig. 4).

Serial changes of serum bilirubin levels in both groups demonstrated almost the same pattern as serum LDH, except that there was no reincrement after week 32 (Fig. $5 A$ ). Serum ALT levels showed a similar pattern to serum bilirubin, although the decrease after week 20 was slightly retarded (Fig. 5B).

Histological changes. All RD rats which had died between weeks 14 and $16(n=33)$ and three of the five RD rats killed at week 15 showed frequently spotty necrosis and enlarged hepatocytes with giant nuclei, suggesting fulminant hepatitis
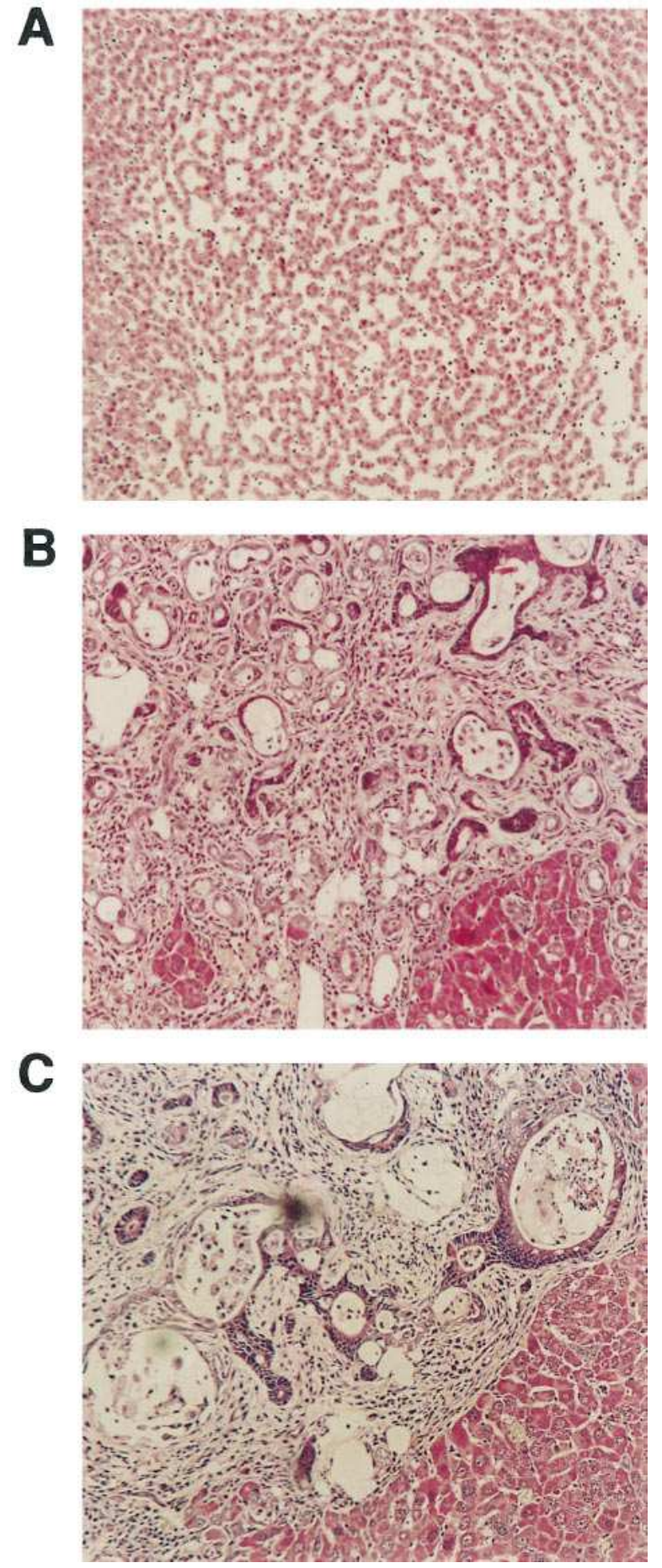

Figure 8. Histological findings of livers obtained from rats fed with $\mathrm{RD}$ and ID at $65 \mathrm{wk}$ after birth. The liver sections were stained with hematoxylin and eosin. Liver sections obtained from all rats in RD group exhibited well-differentiated adenocarcinoma $(A)$. $\times 100$. Cholangiofibrosis was observed in both $\mathrm{RD}(B)$ and ID $(C)$ groups. $\times 200$.

and gross iron accumulation in both nonparenchymal and parenchymal cells; the typical examples are shown in Fig. 6, $A$ and $B$. The two remaining rats killed at week 15 showed moderate necrosis and iron accumulation (data not shown). In all livers obtained from the ID group, on the contrary, very little if any histological changes or iron accumulation were observed 


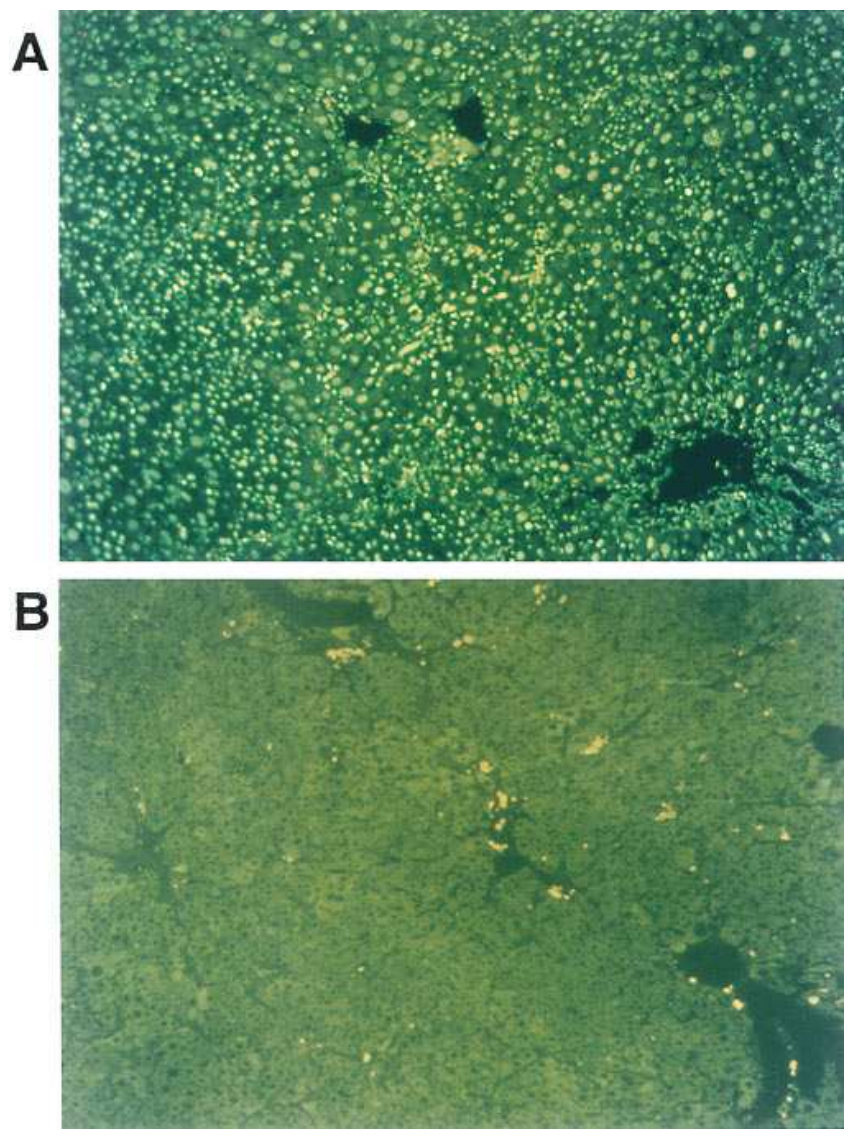

Figure 9. Detection of apoptotic cells in the liver of LEC rats. The liver sections were obtained from rats fed with $\mathrm{RD}(A)$ or ID $(B)$ at $15 \mathrm{wk}$ after birth. Apoptotic cells were stained by using an in situ dUTP nick end-labeling method. $\times 100$.

at week 15 (Fig. 6, $C$ and $D$ ). Furthermore, the degree of fibrosis in the liver of the ID group was far less than that of RD group in the chronic hepatitis phase at week 32 (Fig. 7). Livers obtained from the RD group at week 65 had multiple macronodules, while that of the ID group had none of these lesions, and the mean liver weights of RD and ID group were 17.2 \pm 3.3 $(n=5)$ and $4.3 \pm 0.3$ grams $(n=5)$, respectively. Histological observation at week 65 disclosed that the livers in all rats of the RD group showed well-differentiated adenocarcinoma which was not found at all in the ID group. However, cholangiofibrosis was observed in the livers of both groups, suggesting that it was possibly due to copper toxicity. These typical examples are shown in Fig. 8.

Apoptotic change of hepatocytes. Using in situ dUTP nick end-labeling method, many apoptotic hepatocytes were detected in the liver section of the five rats in the RD group tested at week 15 (Fig. $9 A$ ). In contrast, however, the apoptosis was markedly inhibited in the ID group (Fig. $9 B$ ), indicating that accumulated hepatic iron possibly induced apoptosis via radical formation.

\section{Discussion}

In this report, we have shown that accumulated excess hepatic iron played an important role in the development of fulminant hepatitis and liver cancer in LEC rats.
Copper and iron ions are potentially toxic because they stimulate the generation of radicals through the Fenton reaction $(16,17)$, but they are usually present in tissue bound to metallothionein and ferritin, respectively, and therefore are not toxic $(32,33)$. In LEC rats, both copper and iron may be responsible for the liver injury when they exceed the detoxifying capacity of metallothionein and ferritin.

As for the fulminant hepatitis in LEC rats fed RD, however, iron is more directly involved in its pathogenesis since no increment of hepatic copper was observed in coincidence with a hepatitis episode, while hepatic iron, especially free iron which is more potent to generate oxygen radicals, was clearly elevated during this period. The role of copper in fulminant hepatitis is more likely explained by its indirect action. Hepatic copper storage capacity was limited, and when the limit was exceeded, inorganic copper was released into the circulation, forming a peak at week 15 . These free copper ions in the circulation induced hemolytic anemia, as shown in Fig. 4, possibly by changing erythrocyte membrane permeability or causing osmotic fragility $(22,23)$. Once intravascular hemolysis occurs, $\mathrm{Hb}$ bound to haptoglobin ( $\mathrm{Hb}$-haptoglobin complex) is introduced to the liver via its specific receptors and hepatic iron exceeds the binding capacity of ferritin, resulting in the generation of free iron ions and liver damage. Subsequently, more excess copper ions are released from the damaged liver into the circulation, compounding the condition.

As to the second hemolytic episode occurring in the RD group at week 20 not causing fulminant hepatitis, it may be explained by the fact that ferritin synthesis was sufficiently induced to chelate free iron ions at this stage (14). Moreover, why hemolysis never occurred after 20 wk can be understood by reasoning that free copper was accommodated by newly induced metallothionein (30). Further evidence for the indirect involvement of copper in abrupt hepatic injury via induction of hemolysis was clearly presented by the experiment with the ID group; hemolytic episodes did occur in these rats. The peaks corresponding to the magnitude of hepatic iron (Fig. $2 \mathrm{~B}$ ) were lower in the ID group than in RD group, which could be explained by there being fewer copper ions released from the damaged livers of the ID group. Togashi et al. (34) demonstrated that in LEC rats D-penicillamine, a metal chelating agent, could prevent the development of hepatitis under observation until week 24 , and they concluded that copper ion accumulation in the liver is pathognomonic. However, this could be alternatively explained by proposing that chelation of copper prevented hemolysis and, consequently, hampered excess iron influx to the liver.

A manifestation similar to fulminant hepatitis after hemolytic anemia has also been reported in some WD patients (20). No explanation for this coincidental occurrence of hemolysis and hepatic injury has been made so far. However, on the basis of the LEC rat experiment, we speculate that the accumulation of hepatic iron after hemolysis plays an important role in the liver damage in WD patients as well. The reason for the lower incidence of fatal fulminant hepatitis in WD patients than that seen in LEC rats may be that the basal hepatic iron storage level in LEC rats is considerably higher than in WD patients. Moreover, it may be due to species differences in sensitivity to copper and iron. The fact that the occurrence of liver cancer was completely prevented by the iron-free diet is worthy of remark in relation to iron-induced radical formation which leads to carcinogenesis. However, since iron loading above levels 
seen in the LEC rat does not normally cause hepatoma in rats, and ferrous iron can reduce copper to the cuprous ion which is a more potent generator of hydroxy radicals than ferrous ions (35), iron may be acting synergistically with copper in the carcinogenesis. Further studies may be needed to prove a direct relationship between iron and carcinogenesis in LEC rats.

LEC rats are reported to have various mutations and expression abnormalities including deletion of the P-type ATPase gene (hts gene) which causes human WD, abnormal expression of metallothionein mRNA(36), and abnormal adjustment of expression of transferrin (14). Moreover, we have detected mutations in the class I $A D H$ and $A L D H$-2 genes of LEC rats (37). The essential abnormality of LEC rats which display these genetic aberrations could be due to such mechanisms as genetic instability.

In conclusion, the LEC rat is overloaded with iron and copper due to anomalies in iron and copper metabolism. This model will be important for investigating possible interactions involving iron and copper in hepatocytes and for studying liver pathophysiology of carcinogenesis induced by heavy metals.

\section{Acknowledgments}

We wish to thank Kevin S. Litton for critical review of this manuscript.

This work was supported by Grants-in-Aid for Cancer Research from the Ministry of Education, Science and Culture, Japan.

\section{References}

1. Di Bisceglie, A.M., C. Axiotis, J.H. Hoofnagle, and B.R. Bacon. 1992. Measurement of iron status in patients with chronic hepatitis. Gastroenterology. 102:2108-2113.

2. Lustbalder, E.D., H.W.L. Hann, and B.S. Blumberg. 1983. Serum ferritin as a predictor of host response to hepatitis B virus infection. Science (Wash. DC). 220:423-425.

3. Bassett, M.L., J.W. Halliday, and L.W. Powell. 1986. Value of hepatic iron measurement in early hemochromatosis and determination of the critical iron level associated with fibrosis. Hepatology. 6:24-29.

4. Fargion, S., A.L. Fracanzani, A. Piperno, M. Braga, R. D’Alba, G. Ronchi, and G. Fiorelli. 1994. Prognostic factors for hepatocellular carcinoma in genetic hemochromatosis. Hepatology. 20:1426-1431.

5. Hayashi, H., T. Takikawa, N. Nishimura, M. Yano, T. Isomura, and N. Sakamoto. 1994. Improvement of serum aminotransferase levels after phlebotomy in patients with chronic active hepatitis $\mathrm{C}$ and excess hepatic iron. Am.J. Gastroenterol. 89:986-988.

6. Yoshida, M.C., R. Masuda, M. Sasaki, N. Takeichi, H. Kobayashi, K. Denpo, and M. Mori. 1987. New mutation causing hereditary hepatitis in the laboratory rat. J. Hered. 78:361-365.

7. Li, Y., Y. Togashi, S. Sato, T. Emoto, J.-H. Kang, N. Takeichi, H. Kobayashi, Y. Kojima, Y. Une, and J. Uchino. 1991. Spontaneous hepatic copper accumulation in LEC rats with hereditary hepatitis: a model of Wilson's disease. J. Clin. Invest. 87:1851-1861.

8. Powell, C.J. 1994. Copper-overloaded causes cancer? The LEC rat: a model for human hepatitis, liver cancer, and much more. Hum. Exp. Toxicol. 13:910-912.

9. Schilsky, M.L, R.J. Stockert, and I. Sternlieb. 1994. Pleiotropic effect of LEC mutation: a rodent model of Wilson's disease. Am. J. Physiol. 266:G907G913.

10. Li, Y., Y. Togashi, S. Sato, T. Emoto, J.-H. Kang, N. Takeichi, H. Kobayashi, Y. Kojima, Y. Une, and J. Uchino. 1991. Abnormal copper accumulation in non-cancerous and cancerous liver issues of LEC rats developing hereditary and spontaneous hepatoma. Jpn. J. Cancer Res. 82:490-492.

11. Muramatsu, Y., T. Yamada, M. Miura, T. Sakai, Y. Suzuki, T. Serikawa, R.E. Tanji, and K. Matsumoto. 1994. Wilson's disease gene is homologous to hts causing abnormal copper transport in Long-Evans cinnamon rats. Gastroenterology. 107:1189-1192.

12. Wu, J., J.R. Forbes, H.S. Chen, and D.W. Cox. 1994. The LEC rat has a deletion in the copper transporting ATPase gene homologous to the Wilson disease gene. Nat. Genet. 7:541-545.

13. Thomas, G.R., J.R. Forbes, E.A. Roberts, J.M. Walshe, and D.W. Cox 1995. The Wilson disease gene: spectrum of mutations and their consequences. Nat. Genet. 9:210-217.

14. Kato, J., Y. Kohgo, N. Sugawara, S. Katsuki, N. Shintani, K. Fujikawa, M. Kobune, N. Takeichi, and Y. Niitsu. 1993. Abnormal hepatic iron accumulation in LEC rats. Jpn. J. Cancer Res. 84:219-222.

15. Sugawara, N., T. Ikeda, C. Sugawara, Y. Kohgo, J. Kato, and N. Takeichi. 1992. Regional distribution of copper, zinc and iron in the brain in LongEvans Cinnamon (LEC) rats with a new mutation causing hereditary hepatitis. Brain Res. 588:287-290.

16. Aust, S.D., L.A. Morehouse, and C.E. Thomas. 1985. Role of metals in oxygen radical reactions. J. Free Radical Biol. Med. 1:3-25.

17. Stohs, S.J., and D. Bagchi. 1995. Oxidative mechanisms in the toxicity of metal ions. J. Free Radical Biol. Med. 18:321-326.

18. Yamada, T., K. Sogawa, Y. Suzuki, K. Izumi, T. Agui, and K. Matsumoto. 1992. Elevation of the level of lipid peroxidation associated with hepatic injury in LEC mutant rat. Res. Comm. Chem. Pathol. Pharmacol. 77:121-124.

19. Berger, M., M. de Hazen, A. Nejjari, J. Fournier, J. Guignard, H Pezerat, and J. Cadet. 1993. Radical oxidation reactions of the purine moiety of 2 '-deoxyribonucleosides and DNA by iron-containing minerals. Carcinogenesis. 14:41-46.

20. Roche-Sicot, J., and J.-P. Benhamou. 1977. Acute intravascular hemolysis and acute liver failure associated as a first manifestation of Wilson's disease. Ann. Intern. Med. 86:301.

21. Robitallile, G.A., R.L. Piscatelli, E.J. Majeski, and T.D. Gelehrter. 1977. Hemolytic anemia in Wilson's disease. J. Am. Med. Assoc. 237:2402-2403.

22. Forman, S.J., K.S. Kumar, A.G. Redeker, and P. Hochstein. 1980. Hemolytic anemia in Wilson disease: clinical findings and biochemical mechanisms. Am. J. Hematol. 9:269-275.

23. Deiss, A., G.R. Lee, and G.E. Cartwright. 1971. Hemolytic anemia in Wilson's disease. Ann. Intern. Med. 75:57-65.

24. Drysdale, J.W., and W.N.M. Ramsay. 1965. The separation of ferritin and haemosiderin for studies in the metabolism of iron. Biochem. J. 95:282-288. 25. Baliga, R., N. Ueda, and S.V. Shah. 1993. Increase in bleomycin-detectable iron in ischemia/reperfusion injury to rat kidneys. Biochem. J. 291:901-905.

26. International Committee for Standardization in Haematology. 1967. Recommendations for haemglometry in human blood. Br. J. Haematol. 13(Suppl.):71.

27. Yamanishi, H., S. Iyama, R. Fushimi, and N. Amino. 1996. Interference of ferritin in measurement of serum iron concentrations: comparison by five methods. Clin. Chem. 42:331-332.

28. Makino, T., and J.I. Itoh. 1981. A highly sensitive colorimetric determination of serum copper using alpha, beta, gamma, delta-tetrakis(4- $N$-trimethylaminophenyl)prophine. Clin. Chim. Acta. 111:1-8.

29. Gold, R., M. Schmied, G. Giegerich, H. Bretssschopf, H.P. Hartung, K.V. Toyka, and H. Lassmann. 1994. Differentiation between cellular apoptosis and necrosis by the combined use of in situ tailing and nick translation techniques. Lab. Invest. 71:219-225.

30. Sugawara, N., C. Sugawara, M. Sato, H. Takahashi, and M. Mori. 1992. Excessive accumulation of hepatic copper in LEC rats aged 80 days and 130 days with hepatitis. Pharmacol. Toxicol. 71:321-324.

31. Polterauer, P., R. Rauhs, and H. Puxkandl. 1981. Serum alpha-1-fetoprotein and serum lactate dehydrogenase isoenzymes in liver tumours. Wien. Klin. Wochenschr. 93:227-230.

32. Karin, M. 1985. Metallothioneins: proteins in search of function. Cell. 41:9-10.

33. Munro, H.N., and M.C. Linder. 1978. Ferritin: structure, biosynthesis and role in iron metabolism. Physiol. Rev. 58:317-396.

34. Togashi, Y., Y. Li, J.-H. Kang, N. Takeichi, Y. Fujioka, K. Nagasawa, and H. Kobayashi. 1992. D-penicillamine prevents the development of hepatitis in Long-Evans cinnamon rats with abnormal copper metabolism. Hepatology. 15:82-87.

35. Tachon, P. 1989. Ferric and cupric ions requirement for single-strand breakage by $\mathrm{H}_{2} \mathrm{O}_{2}$. Free Radical Res. Commun. 7:1-10.

36. Kanno, S., Y. Aoki, J.S. Suzuki, N. Takeichi, S. Misawa, and T. Suzuki. 1994. Enhanced synthesis of metallothionein as a possible cause of abnormal copper accumulation in LEC rats. J. Inorg. Biochem. 56:117-125.

37. Nakajima, M., J. Kato, Y. Kohgo, N. Takeichi, and Y. Niitsu. 1994. Point mutation of aldehyde dehydrogenase-2 gene in mutant strains of Long-Evans rats. Alcohol Alcohol. 29:39-43. 